\title{
Recent results on charmonium production in pp collisions
}

\section{H. Hushnud * on behalf of the ALICE Collaboration}

Department of Physics, Aligarh Muslim University

Aligarh, India

E-mail: hushnud.hushnud@cern.ch

The ALICE detector at the LHC has measured the charmonium production at mid and forward rapidity in pp collisions at $\sqrt{s}=5.02 \mathrm{TeV}$. Recent results of the inclusive cross section for $\mathrm{J} / \psi$ and $\psi(2 \mathrm{~S})$ at midrapidity and forward rapidity are reported. Also, the cross section in various differential bins of transverse-momentum $\left(p_{\mathrm{T}}\right)$ for $\mathrm{J} / \psi, \psi(2 \mathrm{~S})$ and the $\psi(2 \mathrm{~S})$-to-J $/ \psi$ cross section ratios are presented. An inclusive cross section in double-differential bins of transverse-momentum $\left(p_{\mathrm{T}}\right)$ and rapidity for $\mathrm{J} / \psi$ at forward rapidity are presented. Finally, the results are compared with several theoretical model calculations.

$22^{\text {nd }}$ Particles and Nuclei International Conference - PANIC2021

5 - 10 September, 2021

Online

${ }^{*}$ Speaker 


\section{Introduction}

In hadronic collisions, the scattering process for the production of a heavy-quark pair can be described with perturbative QCD calculations. On the other hand, the hadronisation of the heavyquark pair is a non-perturbative process which involves long distances and soft momentum scales. Charmonium production measurements in elementary pp collisions at various colliding energies are an important tool for testing various hypothesis, in particular, for investigating the models involving non-perturbative aspects. The charmonium production measurement in pp collisions also constitutes a baseline for the quantification of the quark-gluon plasma formed in nucleus-nucleus collisions and cold nuclear matter effects present in proton-nucleus collisions [1,2].

\section{Experimental setup and data samples}

The ALICE detector [3] at the LHC can measure charmonia down to zero transverse momentum. Measurements are carried out at both central and forward rapidity $(y)$, in the dielectron and dimuon decay channel, respectively. Muons coming from the charmonium decays are reconstructed in the muon spectrometer with $3 \mathrm{Tm}$ field integral, covering the rapidity range $-4<y<-2.5$. The muon spectrometer consists of five tracking stations (MCH) and two trigger stations (MTR). Each tracking station is made out of two planes of cathode pad chambers. The two trigger stations consist of two planes of resistive plate chambers each. These stations provide the necessary event trigger. Charmonium reconstruction in the dielectron decay channel is done in the central barrel with a pseudorapidity range $|\eta|<0.9$. Within the central barrel, inner tracking system (ITS) and time projection chamber (TPC) are used for tracking. The TPC offers the electron identification. The primary and secondary vertex determination relies on the ITS which is useful for the separation of the non-prompt $\mathrm{J} / \psi$ contribution (originated from beauty-hadron decays).

\section{Results}

The $p_{\mathrm{T}}$ and $y$-differential cross section for inclusive charmonium production is given by

$$
\frac{d^{2} \sigma}{d p_{\mathrm{T}} d y}=\frac{N\left(\Delta y, \Delta p_{\mathrm{T}}\right)}{L_{\text {int }} \times B R \times A . \epsilon\left(\Delta y, \Delta p_{\mathrm{T}}\right) \times \Delta p_{\mathrm{T}} \times \Delta y}
$$

where $N\left(\Delta y, \Delta p_{\mathrm{T}}\right)$ is the raw charmonium yield measured in a given $p_{\mathrm{T}}$ and $y$ interval of width $\Delta p_{\mathrm{T}}$ and $\Delta y$, respectively.

The calculated cross sections are compared with theoretical models based on Non-Relativistic QCD (NRQCD) and the Color Glass Condensate (CGC) description of the proton structure for small Bjorken- $x$ gluons from Ma et al. [4]. The NRQCD+CGC calculation provides charmonium production down to very low- $p_{\mathrm{T}}$, whereas at higher transverse moment a NLO collinear factorisation calculation is employed with the same long-distance matrix elements. The Fixed-Order Next-toLeading Logarithm (FONLL) calculations from Cacciari et al. [5] are considered for describing the contribution of non-prompt $\mathrm{J} / \psi$. We point the interested reader also to the results of quarkonium production at $13 \mathrm{TeV}$ presented in Ref [6] as well as the publication [7].

The Figure 1 shows the inclusive $\mathrm{J} / \psi$ cross section in pp collisions at $\sqrt{s}=5.02 \mathrm{TeV}$ for mid (left) and forward rapidity (right). The $p_{\mathrm{T}}$-differential $\mathrm{J} / \psi$ cross section has been compared to: two 

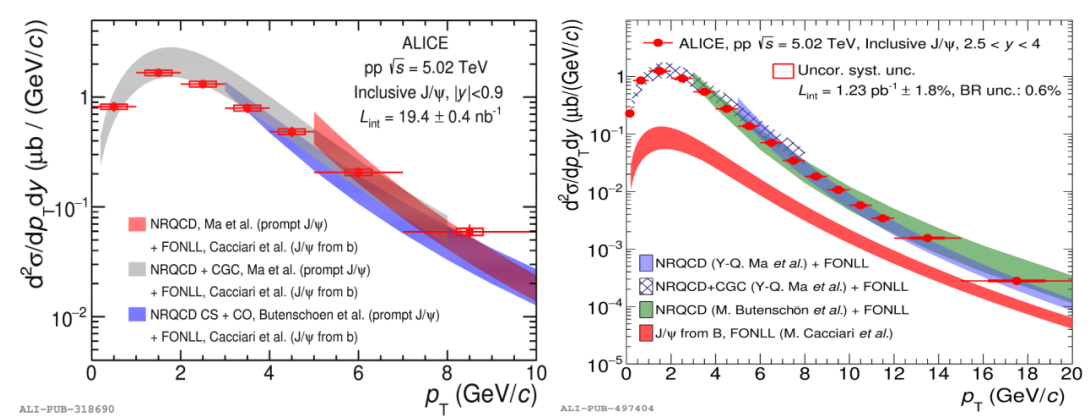

Figure 1: The $p_{\mathrm{T}}$ dependence of $\mathrm{J} / \psi$ production cross section for mid (left) [7] and forward (right) [8] and compared with theoretical models $[4,5,9,10]$.

NLO NRQCD calculations from Butenschön et al. [9] and from Ma et al. [10] and calculations from the NRQCD+CGC calculation. The non-prompt contribution taken from FONLL is also shown. Experimental data are reproduced well by the NRQCD models for $p_{\mathrm{T}}>3 \mathrm{GeV} / c$ for the model from Butenschön et al. and $p_{\mathrm{T}}>5 \mathrm{GeV} / c$ for the model from Ma et al.. All NRQCD calculations, combined with FONLL, are able to describe the production cross sections of inclusive $\mathrm{J} / \psi$ in the corresponding $p_{\mathrm{T}}$ intervals, at mid and forward rapidity.
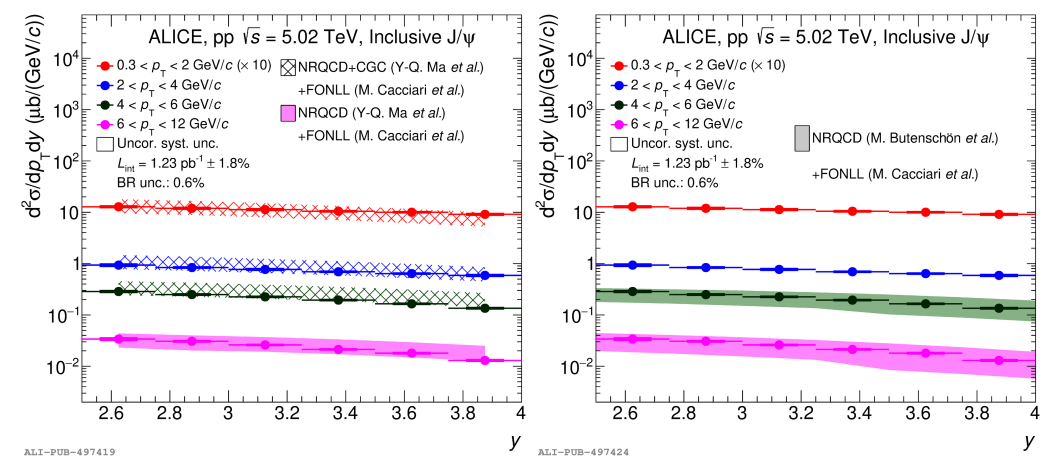

Figure 2: Rapidity dependence of the $J / \psi$ cross section for various $p_{\mathrm{T}}$ ranges [8] and compared to theoretical calculations $[4,5,9,10]$. [Left] The data has been described well over all $p_{\mathrm{T}}$ bins by theoretical models $[4,5,10]$ whereas for bins having $p_{\mathrm{T}}>4 \mathrm{GeV} / c$ by [9] [Right].

Figure 2 displays the inclusive cross section of $\mathrm{J} / \psi$ in double-differential bins of $p_{\mathrm{T}}$ and $y$. The $y$-differential inclusive cross section in various $p_{\mathrm{T}}$ bins are described well by the NRQCD + CGC calculations and the NQRCD calculations from Ma et al.. They describe the data well for the shown distributions.

The inclusive $\psi(2 \mathrm{~S})$ cross section (left) and ratio $\psi(2 \mathrm{~S})$-to-J/ $\psi$ (right), compared with the same theoretical models discussed above have been displayed in Figure 3. A good agreement between experimental data and NRQCD is observed. The calculation from Butenschön et al. for $4<p_{\mathrm{T}}<$ $12 \mathrm{GeV} / c$, and the NRQCD calculation from Ma et al. describe well, except for $5<p_{\mathrm{T}}<6 \mathrm{GeV} / c$, where it overpredicts the data. A comparison of $\psi(2 \mathrm{~S})$-to-J $/ \psi$ production cross section ratio with theoretical models is shown in Figure 3 (right). The ICEM calculation [11] shows a very good 

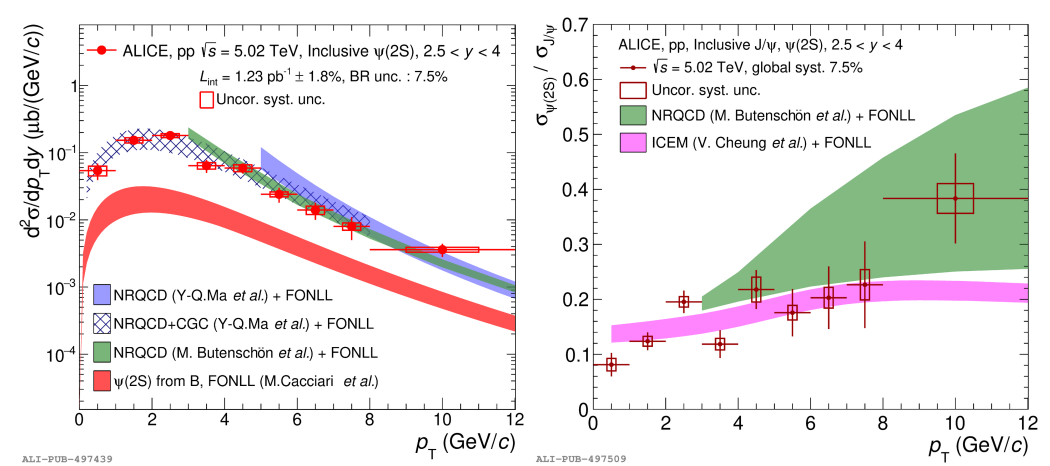

Figure 3: A comparison of $\psi(2 \mathrm{~S})$ production (left) and the ratio $\psi(2 \mathrm{~S})$-to-J $/ \psi$ versus $p_{\mathrm{T}}$ (right) [8] with theoretical models $[4,5,9-11]$.

agreement for this ratio, while the NRQCD calculations from Butenschön et al. seem to just touch the points within the large model uncertainties.

\section{Summary}

The inclusive production cross sections of $\mathrm{J} / \psi$ and $\psi(2 \mathrm{~S})$ have been measured in pp collisions at $\sqrt{s}=5.02 \mathrm{TeV}$ at mid and forward rapidity. Thanks to the large luminosity $\left(1.23 \mathrm{pb}^{-1}\right)$ compared to previous data taking periods, the $\mathrm{J} / \psi$ double-differential cross section as a function of $p_{\mathrm{T}}$ and $y$ has been extracted at forward rapidity. The $\psi(2 \mathrm{~S})$-to-J $/ \psi$ cross section ratio has been measured as a function of $p_{\mathrm{T}}$ at forward rapidity. Model calculations describe well the $p_{\mathrm{T}}$ and $y$-differential measurements. This data set is particularly important as reference for the $\mathrm{Pb}-\mathrm{Pb}$ collision data set recorded at $\sqrt{s}_{\mathrm{NN}}=5.02 \mathrm{TeV}$.

\section{References}

[1] A. Andronic et al., Eur. Phys. J. C76 no.3, (2016) 1534.

[2] A. Rothkopf, Phys. Rept. 858 (2020) 1-117.

[3] ALICE Collaboration, K. Aamodt et al., JINST 33 (2008) S08002.

[4] Y.-Q. Ma and R. Venugopalan, Phys. Rev. Lett. 113 no. 19 (2014) 192301.

[5] M. Cacciari et al., JHEP 10 (2012) 137.

[6] ALICE Collaboration, S. Acharya et al., arXiv:2108.02523 [nucl-ex].

[7] ALICE Collaboration, S. Acharya et al., Eur. Phys. J. C 77 (2017) 392.

[8] ALICE Collaboration, S. Acharya et al., arXiv:2109.15240 [nucl-ex].

[9] M. Butenschön and B. A. Kniehl, Phys. Rev. Lett. 106 (2011) 022003.

[10] Y.-Q. Ma, K. Wang, and K.-T. Chao, Phys. Rev. Lett. 106 (2011) 042002.

[11] V. Cheung and R. Vogt, Phys. Rev. D 98 no. 11, (2018) 114029. 